\title{
POWER SPECTRUM OF A SIGMA-DELTA MODULATOR WITH HEXAGONAL VECTOR QUANTIZATION AND CONSTANT INPUT
}

\author{
Glen Luckjiff and Ian Dobson \\ Electrical and Computer Engineering Dept. \\ University of Wisconsin, Madison WI 53706 USA \\ dobson@engr.wisc.edu
}

\begin{abstract}
A new application of sigma-delta modulation has emerged in soft switching three phase power electronic converters. For this application, a conventional sigma-delta modulator with scalar signals and binary quantizer is generalized to a sigma-delta modulator with vector signals and a truncated hexagonal lattice quantizer. This vector sigma-delta modulator has an interesting output spectrum which is of importance in assessing performance and interpreting results. This paper analytically derives the output spectrum for the case of a generic constant input using ergodic theory and Fourier series on the hexagon.
\end{abstract}

\section{INTRODUCTION}

Three phase soft switching converters are of current interest in power electronics. In particular, the resonant link converter uses zero voltage switching to limit switching losses and attain relatively high switching frequencies [1]. Conventional modulation techniques such as PWM are not applicable to resonant link converters because of the discrete timing of the switchings. However, the sigma-delta $(\Sigma \Delta)$ architecture is well suited to modulation of the resonant link converter. Indeed switching converters can be viewed as analog to digital converters in which an analog reference is coded into a low resolution set of discrete switching states [2]. The relatively high switching frequency of resonant link converters corresponds to a high oversampling ratio with typical values of 20-50. Experimental and simulation results for first and second order $\Sigma \Delta$ hexagonal modulators in a resonant dc link system switching at $100 \mathrm{kHz}$ with a bandwidth of $2 \mathrm{kHz}$ are presented in [2].

To apply the conventional $\Sigma \Delta$ architecture with binary quantization and a single feedback loop to three phase converters requires some generalization. First, the output voltages (line-to-neutral) of a three phase converter with balanced load are limited to a set of seven output vectors (dots

Support in part from NSF PYI grant ECS-9157192 and the UWMadison Industrial \& Economic Development Research program funded by the State of Wisconsin is gratefully acknowledged. in Fig. 2) which form a truncated hexagonal lattice. If we assume a nearest neighbor partition as in the binary quantizer case, the appropriate generalization is the truncated hexagonal lattice quantizer which is well known in communications [3]. Second, all modulator signals are augmented from scalar quantities to vectors and the scalar integrator is replaced by a vector integrator. This generalized modulator is inherently two dimensional and cannot be simply analyzed as the Cartesian product of two scalar modulators.

The output spectrum of a scalar $\Sigma \Delta$ modulator is complicated and it is useful in interpreting results to analytically derive the output spectrum. (The approximate white noise model of quantization error is useful in design but can be misleading for the single-loop case [4].) Powerful techniques from ergodic theory have been deployed by Gray [5, 4], Delchamps [6, 7], and He et al. [8] to derive exact formulas for the spectra of scalar modulators for various inputs. In this paper, we build on their methods to derive the spectrum of a vector $\Sigma \Delta$ modulator with a hexagonal quantizer and a generic constant input.

\section{HEXAGONAL LATTICES}

It is convenient to define the hexagonal lattice in the plane $\mathcal{P}=\left\{(x, y, z)^{t} \in \mathbb{R}^{3} \mid x+y+z=0\right\}$. Define $W: \mathbb{R}^{2} \rightarrow \mathcal{P}$ and $V: \mathcal{P} \rightarrow \mathbb{R}^{2}$ to be

$$
W=\left[\begin{array}{rr}
1 & 0 \\
0 & -1 \\
-1 & -1
\end{array}\right], \quad V=\frac{1}{3}\left[\begin{array}{rrr}
2 & -1 & -1 \\
-1 & 2 & -1
\end{array}\right]
$$

$V W$ is the identity on $\mathbb{R}^{2}$ and $W V$ is the identity on $\mathcal{P}$.

The hexagonal lattice is $\Lambda=\bigcup\left\{W k \mid k \in \mathbb{Z}^{2}\right\}$. The lattice dual [3] to $\Lambda$ is

$\Lambda^{*}=\left\{p \in \mathcal{P}^{*} \mid p \lambda \in \mathbb{Z}\right.$ for all $\left.\lambda \in \Lambda\right\}=\bigcup\left\{q V \mid q \in \mathbb{Z}^{* 2}\right\}$

Vectors in $\mathcal{P}$ or $\Lambda$ are written as column vectors and dual vectors in $\mathcal{P}^{*}$ or $\Lambda^{*}$ are written as row vectors. For example, $n_{a} \in \mathcal{P}$ in Fig. 2 is the column vector $\frac{1}{3}(2,-1,-1)^{t}$. The columns of $W$ generate $\Lambda$ and the rows of $V$ generate $\Lambda^{*}$.

The Voronoi cells (points closest to a given lattice point) of $\Lambda$ are hexagons of side $\sqrt{2 / 3}$. Define the set $H$ to be the 
interior of the Voronoi cell containing 0 , together with a specific choice of 3 non-opposite hexagon sides and 2 opposite hexagon vertices. (These choices ensure that lattice translates of $H$ tile the plane with no overlapping points.) $H$ is the dark central hexagonal region of Fig. 2.

\section{DISCRETE-TIME MODEL}

A discrete time hexagonal $\Sigma \Delta$ modulator is shown in Fig. 1; the signals $\beta, u_{n}, q_{n}$, and $e_{n}$ are vectors in the plane $\mathcal{P} \subset$ $\mathbb{R}^{3}$. The nearest neighbor quantizer $q$ is shown in Fig. 2 . The input $u$ to $q$ is a point in the plane $\mathcal{P}$ and the output $q(u)$ is the nearest to $u$ of the 7 truncated hexagonal lattice points $\left\{0, \pm n_{a}^{\perp}, \pm n_{b}^{\perp}, \pm n_{c}^{\perp}\right\}$ in Fig. 2 .

From Fig. 1, one can write the following difference equations in discrete time $n=0,1,2, \ldots$ :

$$
u_{n+1}=\beta+u_{n}-q\left(u_{n}\right)
$$

where $\beta$ is the constant input, $u_{n}$ is the modulator state, and $q_{n}=q\left(u_{n}\right)$ is the quantizer output at time $n$.

The quantizer error sequence is

$$
e_{n}=e\left(u_{n}\right)=u_{n}-q\left(u_{n}\right)
$$

and the integrator state $u_{n}$ and quantizer output $q_{n}$ can be expressed in terms of of $e_{n}$ :

$$
\begin{aligned}
& u_{n+1}=e_{n}+\beta \\
& q_{n+1}=e_{n}-e_{n+1}+\beta
\end{aligned}
$$

Rewriting (2) in terms of $e_{n}$ yields the nonlinear equations:

$$
\begin{aligned}
e_{0} & =e\left(u_{0}\right) \\
e_{n+1} & =e\left(e_{n}+\beta\right), \quad n=0,1,2 \ldots
\end{aligned}
$$

\section{SOLUTION OF THE DIFFERENCE EQUATION}

Our analysis requires the modulator state $u_{n}, n=0,1,2, \ldots$ to be contained in the no overload region $R$ of the quantizer. $R$ is the shaded region of Fig. 2 consisting of the 7 hexagons closest to zero. $S$ is the star shaped shaded region of Fig. 2. The following sufficient condition for no overload can be shown by induction: If $\beta \in S$ and $e_{0} \in H$, then $e_{n} \in H$ and $u_{n} \in R$ for all $n=0,1,2 \ldots$ We assume $\beta \in S$ and $e_{0} \in H$ and hence no overload for the rest of the paper.

A function $f$ on $\mathcal{P}$ is hexagonally periodic if $f(x+\lambda)=$ $f(x)$ for all $\lambda \in \Lambda$. Define $\square: \mathcal{P} \rightarrow H$ as the identity on $H$ and extend the definition of $\square$ to $\mathcal{P}$ by making $\square$ hexagonally periodic. Let $x, y \in \mathcal{P}$. Then

$$
x+y=x
$$

Since the function $e(u)=u-q(u)$ coincides with $\square$ on the no overload region $R$, the no overload assumption implies that the difference equation (6) can be written as

$$
e_{n+1}=\underline{e_{n}+\beta}
$$

Property (7) can be used to check that the solution to (8) is

$$
e_{n}=e_{0}+n \beta
$$

\section{FOURIER AND ERGODIC RESULTS}

We state results about Fourier analysis and ergodic shifts. Let $f, g: \mathcal{P} \rightarrow \mathbb{C}$ be hexagonally periodic and Lebesgue square integrable on $H$. Then $f(x)=\sum_{p \in \Lambda^{*}} \hat{f}_{p} e^{i 2 \pi p x}$ where the equality is interpreted in the $\mathcal{L}^{2}$ sense and the Fourier coefficients are $\hat{f}_{p}=\frac{1}{|H|} \int_{H} f(s) e^{-i 2 \pi p s} d s .|H|=$ $\sqrt{3}$ is the area of $H$. Parseval's formula is

$$
\frac{1}{|H|} \int_{H} f(s) g^{*}(s) d s=\sum_{p \in \Lambda^{*}} \hat{f}_{p} \hat{g}_{p}^{*}
$$

These Fourier results can be obtained either as sketched in appendix $A$ or as a particular case of harmonic analysis on compact Abelian groups [9].

Identify points of $\mathcal{P}$ differing by vectors in $\Lambda$ to define $\mathcal{H}=\mathcal{P} / \Lambda=\{x+\Lambda \mid x \in \mathcal{P}\} . \mathcal{H}$ is a compact Abelian group. A function $f: H \rightarrow \mathbb{C}$ lifts to a function $\tilde{f}: \mathcal{H} \rightarrow \mathbb{C}$ if $\tilde{f}(x+\Lambda)=f(x)$ for all $x$. Lemma 1 gives conditions on the input $\beta$ for the dynamics (9) to induce a uniquely ergodic shift on $\mathcal{H}$ so that time averages of a function $f$ can be evaluated as an integral over $H$ :

Lemma 1. Let $\beta \in \mathcal{P}$ be such that the only $p \in \Lambda^{*}$ with $p \beta \in \mathbb{Z}$ is $p=0$. Let $f: H \rightarrow \mathbb{R}$ have a continuous lifting $\tilde{f}: \mathcal{H} \rightarrow \mathbb{R}$ Then for all $e_{0} \in \mathcal{P}$,

$$
\lim _{L \rightarrow \infty} \frac{1}{L} \sum_{n=0}^{L-1} f\left(\underline{\underline{e_{0}+n \beta}}\right)=\frac{1}{|H|} \int_{H} f(s) d s
$$

Corollary 1. The result of Lemma 1 extends to functions $f: H \rightarrow \mathbb{R}$ for which there exist sequences of functions $\bar{f}_{k}$, $\underline{f}_{k}, k=1,2, \ldots$ with continuous liftings such that $\underline{f}_{k}(x) \leq$ $f(x) \leq \bar{f}_{k}(x)$ for all $x$ and $\left|\underline{f}_{k}-\bar{f}_{k}\right|_{\infty} \rightarrow 0$ as $k \rightarrow \infty$.

Lemma 1 can be obtained from standard results on the torus [10] as indicated in appendix A.

\section{SPECTRAL ANALYSIS}

We make the generic assumption that the input $\beta$ satisfies the condition of Lemma 1. (If, exceptionally, $\beta$ does not satisfy the condition of Lemma 1 then either $e_{n}$ is periodic or $e_{n}$ is aperiodic and constrained to a submanifold of $H$.)

The mean of the noise process is, using (9),

$$
\bar{e}=\lim _{L \rightarrow \infty} \frac{1}{L} \sum_{n=0}^{L-1} e_{n}=\lim _{L \rightarrow \infty} \frac{1}{L} \sum_{n=0}^{L-1} \underline{e_{0}+n \beta}
$$


Applying Lemma 1 to each component of the identity map on $H$ yields

$$
\vec{e}=\frac{1}{|H|} \int_{H} s d s=0
$$

so that $\bar{e}$ is well defined and independent of $e_{0}$.

The autocorrelation matrix of the noise process is

$$
R_{e}(k)=\lim _{L \rightarrow \infty} \frac{1}{L} \sum_{n=0}^{L-1} e_{n} \otimes e_{n+k}
$$

where $\otimes$ denotes outer product. Using (9) and (7) gives

$$
R_{e}(k)=\lim _{L \rightarrow \infty} \frac{1}{L} \sum_{n=0}^{L-1} e^{e_{0}+n \beta} \otimes e^{e_{0}+n \beta}+k \beta
$$

Let $f: H \rightarrow \mathcal{P} \times \mathcal{P}$ be defined by $f(s)=s \otimes\langle s+k \beta$. Each component of $f$ is continuous except on several line segments and satisfies the conditions of Corollary 1 . Hence

$$
R_{e}(k)=\frac{1}{|H|} \int_{H}(s) \otimes \sqrt{s+k \beta} d s
$$

Partition $\Lambda^{*}=\Lambda^{t} \cup\left(n_{a}^{t}+\Lambda^{t}\right) \cup\left(-n_{a}^{t}+\Lambda^{t}\right)$. Using coordinates $p=\left(p_{a}, p_{b}, p_{c}\right)$, define $\sigma$ and $\Pi$ on $\Lambda^{*}$ by

$$
\sigma(p)=\left\{\begin{array}{cl}
0 & \text { if } p \in \Lambda^{t}, p_{a} p_{b} p_{c} \neq 0 \text { or } p=0 \\
2 \pi / \sqrt{3} & \text { if } p \in \Lambda^{t}, p_{a} p_{b} p_{c}=0 \text { and } p \neq 0 \\
1 & \text { if } p \in n_{a}^{t}+\Lambda^{t} \\
-1 & \text { if } p \in-n_{a}^{t}+\Lambda^{t}
\end{array}\right.
$$

$\Pi(p)=$ product of nonzero elements of $\left\{p_{a}, p_{b}, p_{c}\right\}, p \neq 0$ and $\Pi(0)=1$. Let $c_{p}$ be the Fourier coefficients of $\square$ :

$$
c_{p}=\frac{1}{|H|} \int_{H}(s) e^{-i 2 \pi p s} d s
$$

Appendix B computes $c_{p}$ as

$$
c_{p}=\frac{-i \sigma(p) p^{t}}{4 \sqrt{3} \pi^{2} \Pi(p)}
$$

The Fourier coefficients of $f(x)=x+k \beta$ are $c_{p} e^{i 2 \pi p \beta k}$. Each entry of the outer product in (12) can be regarded as an inner product of functions over $H$ and applying Parseval's formula (10) to each entry gives

$$
R_{e}(k)=\sum_{p \in \Lambda^{*}} c_{p}^{*} \otimes c_{p} e^{i 2 \pi p \beta k}
$$

The Bohr-Fourier series (15) implies that the quantization error spectral density matrix $S_{e}$ is purely discrete having amplitudes $c_{p}^{*} \otimes c_{p}$ at frequencies $\langle p \beta\rangle$ for $p \in \Lambda^{*}$ :

$$
S_{e}(\omega)=\sum_{p \in \Lambda^{*}} c_{p}^{*} \otimes c_{p} \delta(\omega-\langle p \beta\rangle)
$$

where $\langle p \beta\rangle$ denotes the fractional part of $p \beta$. The quantizer output $q_{n}$ is obtained by differencing $e_{n}$ and adding $\beta$ according to (5). Hence the output spectral density matrix $S_{q}(\omega)=\beta \otimes \beta \delta(\omega)+4 \sin ^{2}(\pi \omega) S_{e}(\omega)$ and, finally,

$$
\begin{aligned}
S_{q}(\omega)= & \beta \otimes \beta \delta(\omega)+ \\
& \sum_{p \in \Lambda^{*}} \frac{\sin ^{2}(\pi \omega) \sigma^{2}(p)}{12 \pi^{4} \Pi^{2}(p)} p \otimes p \delta(\omega-\langle p \beta\rangle)
\end{aligned}
$$

\section{APPENDIX A}

We transform standard Fourier and ergodic results from the square $[0,1)^{2}$ to $H$. Relate coordinates $x^{\prime}$ on $[0,1)^{2}$ to coordinates $x$ on $H$ by $x^{\prime}=\langle V x\rangle$ and $x=\langle\overline{W x}\rangle$. Relate coordinates $p$ on $\Lambda^{*}$ to coordinates $p^{\prime}$ on $\mathbb{Z}^{* 2}$ by $p^{\prime}=p W$. Suppose $f^{\prime} \in \mathcal{L}^{2}\left([0,1)^{2}\right)$ and let $f=f^{\prime} \circ\langle\rangle \circ V \in \mathcal{L}^{2}(H)$. Then $f^{\prime}\left(x^{\prime}\right)=f^{\prime}(\langle V x\rangle)=f(x)$, $d x=|H| d x^{\prime}$ and $\left\langle p^{\prime} x^{\prime}\right\rangle=\langle p W\langle V x\rangle\rangle=\langle p x\rangle$. Write $(f, g)=$ $\frac{1}{[H \mid} \int_{H} f(x) g^{*}(x) d x$ and $\left(f^{\prime}, g^{\prime}\right)=\int_{[0,1)^{2}} f^{\prime}\left(x^{\prime}\right) g^{\prime *}\left(x^{\prime}\right) d x^{\prime}$. Then $(f, g)=\left(f^{\prime}, g^{\prime}\right)$ and $\hat{f}_{p}=\left(f, e^{-i 2 \pi p x}\right)=\left(f^{\prime}, e^{-i 2 \pi p^{\prime} x^{\prime}}\right)=\hat{f}_{p^{\prime}}^{\prime}$. Moreover, $f(x+y))=f^{\prime}(\langle V x+y\rangle)=f^{\prime}(\langle V(x+y)\rangle)$ $=f^{\prime}\left(\left\langle x^{\prime}+y^{\prime}\right\rangle\right)$. Hence the following results can be transformed to the results of section 5:

Fourier analysis on $[0,1)^{2}[11]: \mathcal{L}^{2}\left([0,1)^{2}\right)$ is a Hilbert space with inner product $\left(f^{\prime}, g^{\prime}\right)$. $\left\{e^{i 2 \pi p^{\prime} x^{\prime}} \mid p^{\prime} \in \mathbb{Z}^{* 2}\right\}$ is a complete orthonormal basis. $f^{\prime}\left(x^{\prime}\right)=\sum_{p^{\prime} \in \mathbf{Z}^{* 2}} \hat{f}_{p^{\prime}}^{\prime} e^{i 2 \pi p^{\prime} x^{\prime}}$ where the equality is interpreted in the $\mathcal{L}^{2}$ sense and $\hat{f}_{p^{\prime}}^{\prime}=\left(f^{\prime}, e^{-i 2 \pi p^{\prime} x^{\prime}}\right)$. The Parseval formula $\left(f^{\prime}, g^{\prime}\right)=\sum_{p^{\prime} \in \mathbf{Z} * 2} \hat{f}_{p^{\prime}}^{\prime} \hat{g}_{p^{\prime}}^{\prime *}$ holds.

Manẽ [10] (thm. II 3.2, prop. II 2.7, thm. I 9.2) implies: Let $\beta^{\prime} \in \mathbb{R}^{2}$ be such that the only $p^{\prime} \in \mathbb{Z}^{* 2}$ with $p^{\prime} \beta^{\prime} \in \mathbb{Z}$ is $p^{\prime}=0$. Let $\tilde{f}^{\prime}: \mathbb{R}^{2} / \mathbb{Z}^{2} \rightarrow \mathbb{R}$ be continuous. Let $\pi$ be the canonical projection $\mathbb{R}^{2} \rightarrow \mathbb{R}^{2} / \mathbb{Z}^{2}$. Then translation by $\pi \beta^{\prime}$ is a uniquely ergodic shift on $\mathbb{R}^{2} / \mathbb{Z}^{2}$ and, for all $e_{0}^{\prime} \in \mathbb{R}^{2}$,

$$
\lim _{L \rightarrow \infty} \frac{1}{L} \sum_{n=0}^{L-1} \tilde{f}^{\prime}\left(\pi\left\langle e_{0}^{\prime}+n \beta^{\prime}\right\rangle\right)=\int_{\mathbf{R}^{2} / \mathbf{Z}^{2}} \tilde{f}^{\prime}\left(s^{\prime}\right) d s^{\prime}
$$

(Note that $\pi\left(e_{0}^{\prime}+n \beta^{\prime}\right)=\pi\left\langle e_{0}^{\prime}+n \beta^{\prime}\right\rangle$.) Suppose that $f^{\prime}$ : $[0,1)^{2} \rightarrow \mathbb{R}$ lifts to $\tilde{f}^{\prime}$ so that $f^{\prime}=\tilde{f}^{\prime} \circ \pi$. Then

$$
\lim _{L \rightarrow \infty} \frac{1}{L} \sum_{n=0}^{L-1} f^{\prime}\left(\left\langle e_{0}^{\prime}+n \beta^{\prime}\right\rangle\right)=\int_{[0,1)^{2}} f^{\prime}\left(s^{\prime}\right) d s^{\prime}
$$

\section{APPENDIX B: Compute Fourier coefficients $c_{p}$}

It is convenient to define vectors $n$ and $n^{\perp}$ (see Fig. 2):

$$
\begin{aligned}
& {\left[n_{a}\left|n_{b}\right| n_{c}\right]=\frac{1}{3}\left[\begin{array}{ccc}
2 & -1 & -1 \\
-1 & 2 & -1 \\
-1 & -1 & 2
\end{array}\right]} \\
& {\left[n_{a}^{\perp}\left|n_{b}^{\perp}\right| n_{c}^{\perp}\right]=\left[\begin{array}{ccc}
0 & 1 & -1 \\
-1 & 0 & 1 \\
1 & -1 & 0
\end{array}\right]}
\end{aligned}
$$

Also define $n_{-s}=-n_{s}$ and $n_{-s}^{\perp}=-n_{s}^{\perp}$ for $s=a, b, c$. 
Equation (13) implies $c_{0}=0$. Now we compute $c_{p}$ for $p \neq 0$. Define $a: \mathcal{P}^{*} \rightarrow \mathbb{R}$ by $a(p)=-\int_{\mathcal{H}} e^{-i 2 \pi p x} d x$. Then

$$
c_{p}=\frac{1}{i 2 \pi|\mathcal{H}|}\left(D_{p} a(p)\right)^{t}
$$

and the problem becomes finding an expression for $a(p)$ :

$$
\begin{aligned}
a(p) & =\frac{p}{i 2 \pi p p^{t}} \int_{\mathcal{H}}\left(D_{x} e^{-i 2 \pi p x}\right)^{t} d x \\
& =\frac{p}{i 2 \pi|p|^{2}\left|n^{\perp}\right|} \oint_{\partial \mathcal{H}} e^{-i 2 \pi p \ell} n_{\ell}^{\perp} d \ell \\
& =\frac{|n|}{i 2 \pi|p|^{2}\left|n^{\perp}\right|} \sum_{s \in \pm\{a, b, c\}} p n_{s}^{\perp} \int_{-\frac{1}{2}}^{\frac{1}{2}} e^{-i 2 \pi p \sigma_{s}(t)} d t
\end{aligned}
$$

where $\sigma_{s}(t)=\frac{1}{2} n_{s}^{\perp}+n_{s} t, t \in[-1 / 2,1 / 2]$ parameterizes the hexagon edges. Then letting $p_{s}=p n_{s}$ and $p_{s}^{\perp}=p n_{s}^{\perp}$ gives

$$
\begin{aligned}
a(p) & =\frac{-|n|}{\pi\left|n^{\perp}\right||p|^{2}} \sum_{s \in\{a, b, c\}} p_{s}^{\perp} \int_{-\frac{1}{2}}^{\frac{1}{2}} \sin \left(2 \pi p \sigma_{s}(t)\right) d t \\
& =\frac{-1}{\pi^{2} \sqrt{3}|p|^{2}} \sum_{s \in\{a, b, c\}} \frac{p_{s}^{\perp}}{p_{s}} \sin \left(\pi p_{s}\right) \sin \left(\pi p_{s}^{\perp}\right)
\end{aligned}
$$

Substituting (18) in (17), differentiating, and evaluating at $p \in$ $\Lambda^{*}$ (then $p_{s}^{\perp} \in \mathbb{Z}$ and $\sin \left(\pi p_{s}^{\perp}\right)=0$ ) yields

$$
c_{p}=\frac{i}{6 \pi^{2}|p|^{2}} \sum_{s \in\{a, b, c\}} \frac{p_{s}^{\perp}}{p_{s}} \sin \left(\pi p_{s}\right) \cos \left(\pi p_{s}^{\perp}\right) n_{s}^{\perp}
$$

In the case $p \in \Lambda^{t}-\{0\}, p_{s} \in \mathbb{Z}$ and $\sin \left(\pi p_{s}\right) /\left(\pi p_{s}\right)=0$ and $c_{p}=0$ except when $p_{s}=0$. When $p_{s}=0, p=k n_{s}^{1^{t}}$, $k \in \mathbb{Z}, p_{s}^{\perp}=2 k$ and (14) follows from (19).

To simplify (19) in the case $p \in \Lambda^{*}-\Lambda^{t}$, consider $f_{s}: \Lambda^{*} \rightarrow$ $\mathbb{R}$ defined by

$$
f_{s}(p)=2 \sin \left(\pi p_{s}\right) \cos \left(\pi p_{s}^{\perp}\right)=\sin \left(\pi p n_{s}^{+}\right)+\sin \left(\pi p n_{s}^{-}\right)
$$

where $n_{s}^{ \pm}=n_{s} \pm n_{s}^{\perp}$. Notice that $n_{s}^{ \pm} \in 2 \Lambda^{* t}$. Let $\lambda \in \Lambda$. Then

$$
f_{s}\left(p+\lambda^{t}\right)=\sin \left(\pi\left(p+\lambda^{t}\right) n_{s}^{+}\right)+\sin \left(\pi\left(p+\lambda^{t}\right) n_{s}^{-}\right)=f_{s}(p)
$$

since $n_{s}^{ \pm} \in 2 \Lambda^{* t}$ implies that $\lambda^{t} n_{s}^{ \pm}$is an even integer. This periodicity of $f_{s}$ implies that $f_{s}$ is constant on each of $n_{\mathrm{a}}^{t}+\Lambda^{t}$ and $-n_{a}^{t}+\Lambda^{t}$. The respective constants can be directly calculated: $f_{s}\left( \pm n_{a}^{t}\right)= \pm \sqrt{3}$ for $s=a, b, c$. Then $f_{s}(p)=\sigma(p) \sqrt{3}$ and

$$
c_{p}=\frac{i \sigma(p)}{4 \sqrt{3} \pi^{2}|p|^{2}} \sum_{s \in\{a, b, c\}} \frac{p_{s}^{\perp}}{p_{s}} n_{s}^{\perp} ; p \in \Lambda^{*}-\Lambda^{t} .
$$

Now (14) follows by algebra and $p_{a}+p_{b}+p_{c}=0$.

\section{REFERENCES}

[1] D.M. Divan, "The resonant dc link converter," in Proc. Ind. Appl. Soc., Denver, 1986, pp. 648-656.

[2] G. Luckjiff and I. Dobson, "Interpolative sigma-delta modulators for high frequency power electronic applications,' in Proc. Power Electronics Specialists Conf., Atlanta, June 1995, pp. 444-449.

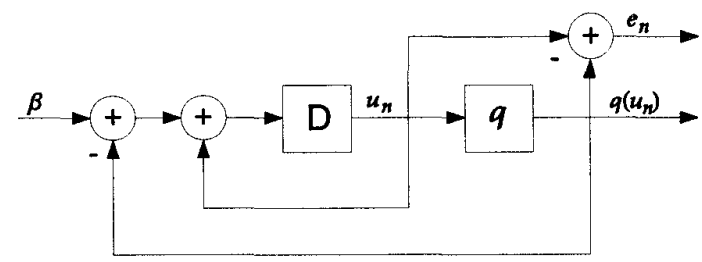

Figure 1: Discrete-time hexagonal $\Sigma \Delta$ modulator. $D$ : unit delay. $q$ : hexagonal quantizer.

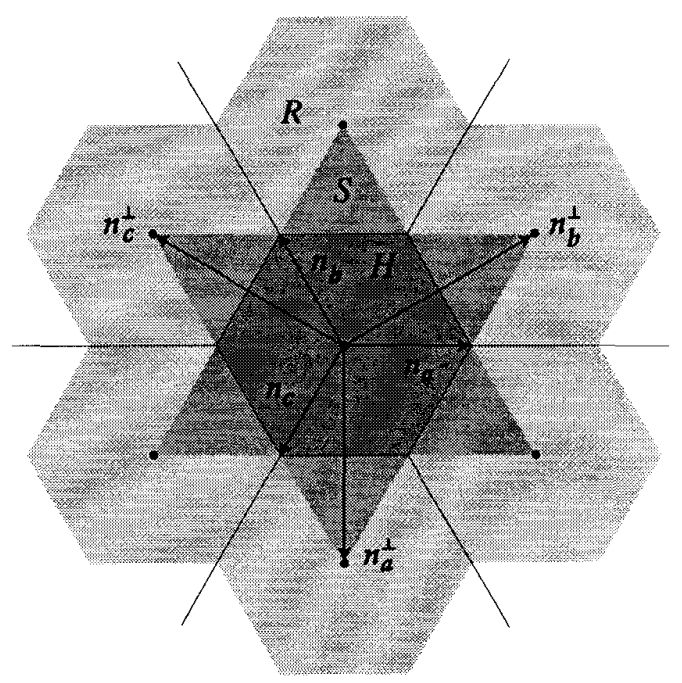

Figure 2: Hexagonal quantizer $q$ and regions $H, S$, and $R$.

[3] J.H. Conway and N.J.A. Sloane, Sphere Packings, Lattices and Groups, Springer-Verlag, NY, second edition, 1993.

[4] R.M. Gray, "Quantization noise spectra," IEEE Trans. Inform. Theory, vol. 36, no. 6, pp. 1220-1244, Nov. 1990.

[5] R.M. Gray, "Spectral analysis of quantization noise in a single-loop sigma-delta modulator with dc input," IEEE Trans. Commun., vol. 37, no. 6, pp. 588-599, June 1989.

[6] D.F. Delchamps, "Exact asymptotic statistics for sigmadelta quantization noise," in Proc. Allerton Conf. Commun., Contr, and Computing, Urbana, Oct. 1990, pp. 703-712.

[7] D.F. Delchamps, "Spectral analysis of sigma-delta quantization noise," in Proc. Inform. Sciences and Syst., Johns Hopkins University, Baltimore, Mar. 1990.

[8] N. He, F. Kuhlmann, and A. Buzo, "Double-loop sigmadelta modulation with dc input," IEEE Trans. Commun., vol. COM-38, pp. 106-114, Apr. 1990.

[9] W. Rudin, Fourier Analysis on Groups, Wiley, NY, 1962.

[10] R. Mãné, Ergodic Theory and Differentiable Dynamics, Springer-Verlag, NY, 1987.

[11] A. Zygmund, Trigonometric Series, Cambridge Univ. Press, second edition, 1959. 